\title{
Les émissions de gaz à effet de serre (GES)
}

Les émissions de $\mathrm{CO}_{2}$ par habitant varient considérablement selon les pays. Le record appartient au Qatar avec près de 44 tonnes de $\mathrm{CO}_{2}$ par habitant et par an. L'ensemble des pays du Golfe ont des émissions qui varient autour de 20 tonnes par habitant. Viennent ensuite les États-Unis (17 tonnes). La plupart des pays d'Europe occidentale émettent entre 7 et 9 tonnes. La France, la Suède et la Suisse sont à un peu plus de 5 tonnes grâce à l'énergie hydroélectrique et au nucléaire, c'est-à-dire un peu moins que la Chine (6,6 tonnes). Compte tenu de sa population, la Chine est le principal émetteur de $\mathrm{CO}_{2}$, suivie par les États-Unis puis l'Union européenne (source CDIAC 2011).

Pour l'année 2013, les émissions de GES en France ont été de 491,1 millions de tonnes d'équivalent $\mathrm{CO}_{2}$, en diminution de $11 \%$ par rapport à 1990. Les transports représentaient 132,6 Mt soit $27 \%$ des émissions, en hausse de 9,5\% par rapport à 1990, mais en légère baisse par rapport aux années 2000 à 2007. La quasi-totalité de ces émissions (près de $94 \%$ ) est due aux transports routiers, dont plus de la moitié aux véhicules particuliers. L’industrie manufacturière représentait 63,7 Mt en 2013 (13\% des émissions) en baisse de $26 \%$ par rapport à 1990, ce qui traduit pour l'essentiel la baisse des activités 
industrielles, notamment dans des industries à forte intensité énergétique comme la sidérurgie. Le secteur résidentiel et tertiaire a émis la même année 86,8 Mt (18\% des émissions), à peine moins qu'en 1990. Les émissions de l'agriculture en représentaient 79,5 Mt (16,2 \% du total), en baisse de $8 \%$ par rapport à 1990 , partagées à peu près à égalité entre l'élevage, en particulier des ruminants, et les engrais. Le reste des émissions provenait des industries de l'énergie (électricité, raffinage, chauffage urbain...) pour 41,1 Mt (soit 8,4\% du total), en baisse de $17 \%$ par rapport à 1990. En sens contraire, l'utilisation des terres, leur changement, et la forêt ont constitué un puits qui a permis l'absorption de 44,3 Mt de GES.

Le rapport de l'American Meteorological Society cité plus haut indique que la concentration dans l'atmosphère des gaz à effet de serre a continué à augmenter : pour la première fois, la concentration en $\mathrm{CO}_{2}$ à l'observatoire de Mauna Loa a dépassé 400 ppm en 2015. 